\title{
Numerically and parallel scalable TFETI based algorithms for quasistatic contact problems of mechanics
}

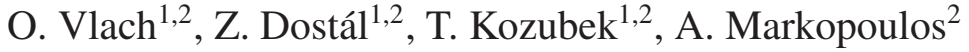 \\ \& T. Brzobohatý ${ }^{2}$ \\ ${ }^{1}$ Faculty of Electrical Engineering, VŠB-Technical University of Ostrava, \\ Czech Republic \\ ${ }^{2}$ Centre of Excellence IT4I, VŠB-Technical University of Ostrava, \\ Czech Republic
}

\begin{abstract}
This paper deals with the solution of the discretized quasistatic 3D Signorini problems with local Coulomb friction. After a time discretization we obtain a system of static contact problems with Coulomb friction. Each of these problems is decomposed by the TFETI domain decomposition method used in auxiliary contact problems with Tresca friction. The algebraic formulation of these problems in $3 \mathrm{D}$ leads to the quadratic programing with equality constraints together with box and separable quadratic constraints. For the solution we used the scalable algorithm SMALSE developed at our department. The efficiency of the method is demonstrated by results of numerical experiments with parallel solution of 3D contact problems of elasticity.
\end{abstract}

\section{Introduction}

Contact problems represent a branch of mechanics of solids which analyzes the behavior of loaded, deformable bodies being in a mutual contact. If the system of bodies includes "floating" bodies, the resulting stiffness matrices from the discretization of such bodies are positive semi-definite. Moreover the occurrence of non-penetration and friction conditions implies the highly non-linear behavior of resulting problems. Both this phenomena depend on time so as the applied forces. If however applied forces vary only slowly in time, inertia of the system 
can be neglected and one can use a quasistatic approximation (Eck et al. [1]). This typically arises in geomechanics (modelling of a movement of tectonic plates, prediction of earthquakes).

Using finite-difference approximation of the time derivative we arrive at a sequence of static contact problems with Coulomb friction, whose solutions are defined by using a fixed point approach. Each iterative step is represented by an auxiliary contact problem with a given slip bound (Tresca model of friction). Since its formulation is given by a variational inequality of the second kind, after discretization we obtain a convex non-smooth constrained minimization problem for a discrete total potential energy function. To increase the efficiency of the quadratic part of the minimized problem we used the TFETI method, a variant (see Dostál et al. [2] and [3]) of FETI domain decomposition methods framework (Farhat et al. [4]). Introducing the additional Lagrange multipliers one can release the non-penetration conditions and transform the frictional into a smooth one. Eliminating the displacement field we arrive at a quadratic minimization problem with box and separated quadratic inequality constraints.

For solving of such minimization problem we use the SMALSE algorithm (Dostál and Kozubek [5] and Dostál and Kučera [6]) with an error bound in terms of bounds on the spectrum of the Hessian to the quadratic cost function, independent of the conditioning of the constraints. Moreover if the problem has sparse enough representation of the Hessian, it simply follows that the cost of the solution is proportional to the number of unknowns.

This proceeding extends the results of Haslinger et al. [7] and [8] from unilateral 2D and 3D problems to multibody contact case.

\section{Formulation of the quasistacic 3D contact problem with Coulomb friction}

At first we briefly introduce the multibody quasistatic problem with Coulomb friction. The $n_{\Omega}$ isotropic elastic bodies are represented by the domains $\Omega^{p} \in \mathbb{R}^{3}$, with a sufficiently smooth boundary $\Gamma^{p}, p=1, \ldots, n_{\Omega}$. Suppose that each $\Gamma^{p}$ consists of three disjoint parts $\Gamma_{U}^{p}, \Gamma_{F}^{p}$ and $\Gamma_{C}^{p}$ and consider zero displacements on $\Gamma_{U}^{p}$ and given forces $F^{p}$. The part $\Gamma_{C}^{p}$ denotes the part of $\Gamma^{p}$ that may get into contact with some other body in a time interval $\left[0, T_{0}\right]$ and therefore the nonpenetration and Coulomb friction conditions have to be prescribed. In particular, we denote by $\Gamma_{C}^{p q}$ the part of $\Gamma_{C}^{p}$ that can be, in the solution, in contact with the body $\Omega^{q}$. For convenience, we introduce the index set of contact interfaces

$$
\mathcal{I}_{C}=\left\{(p, q), p, q=1, \ldots, s: p<q \text { and meas } \Gamma_{C}^{p q}>0\right\} .
$$

One can read more about the formulation for unilateral contact in $[8,9]$. Our notation of the multibody case is similar to the prepared article [3] on transient problems.

By the weak formulation of the quasistatic contact problem with local Coulomb law of friction we mean the following problem formulated in terms of the 
displacement vector $u$ and the normal contact stress $\lambda_{N}$ :

$$
\begin{aligned}
& \text { Find } u \in W^{1,2}\left(0, T_{0}, \mathcal{V}\right), \lambda_{N} \in W^{1,2}\left(0, T_{0}, \mathcal{M}\right) \text { such that } \\
& u \in \mathcal{K} \quad \text { for a.a. } t \in\left[0, T_{0}\right], \quad u(0)=u_{0} \quad \text { in } \Omega \\
& a(u, v-\dot{u})+j\left(\lambda_{N}, v\right)-j\left(\lambda_{N}, \dot{u}\right) \geq L(v-\dot{u})+ \\
& +\sum_{(p, q) \in \mathcal{I}_{C}}\left\langle\lambda_{N}^{p q},\left[v_{N}^{p q}-\dot{u}_{N}^{p q}\right]\right\rangle \quad \forall v \in \mathcal{V} \text { and a.a. } t \in\left[0, T_{0}\right] \\
& \left\langle\lambda_{N}, z_{N}-u_{N}\right\rangle \geq 0 \quad \forall z \in \mathcal{K} \text { and a.a. } t \in\left[0, T_{0}\right],
\end{aligned}
$$

where [ ] is the jump of the displacement across $\Gamma_{C}^{p q}$ and \langle\rangle is the appropriate duality pairing.

$$
\begin{aligned}
a(u, v) & :=\sum_{p=1}^{n_{\Omega}} \int_{\Omega^{p}} c_{i j k l}^{p} \varepsilon_{i j}\left(u^{p}\right) \varepsilon_{k l}\left(v^{p}\right) d \Omega, \quad j\left(\lambda_{N}, v\right):=-\sum_{(p, q) \in \mathcal{I}_{C}}\left\langle\mathcal{F} \lambda_{N}^{p q},\left\|\left[v_{T}^{p q}\right]\right\|\right\rangle \\
L(v) & :=\sum_{p=1}^{n_{\Omega}} \int_{\Omega^{p}} F_{i}^{p} v_{i}^{p} d \Omega+\int_{\Gamma_{p}} P_{i}^{p} v_{i}^{p} d \Gamma
\end{aligned}
$$

where $\lambda_{N}$ is equal to the normal contact stress on $\Gamma_{c}$.

After discretization of time ( $n_{t s}$ timesteps) and space and substitution of the difference instead of time derivation will be the quasistatic problem (1) approximated by the sequence of static contact problems with Coulomb friction. Only the small change will appear in the nonsmooth friction term formulation which is in each timestep corrected from the previous timestep solution. For details see Haslinger et al. [8]. The variational formulation of Coulomb friction is the implicit variational inequality due to the occurrence of the term $\lambda_{N}$ in the nonsmooth part of it. To deal with this we used the alternative formulation based on the fixed point approach, where the sequence of auxiliary Tresca friction problems with previously mentioned correction is solved. It turned out to be efficient from the numerical point of view.

The discretized Tresca friction problem is described as a solution $\mathbf{u}$ of the problem

$$
\left.\begin{array}{l}
\text { For given } \mathbf{g} \in \mathbb{R}_{-}^{n_{N}} \text { find } \mathbf{u} \in \mathbb{K} \text { such that } \\
J(\mathbf{u}) \leq J(\mathbf{v}) \quad \forall \mathbf{v} \in \mathbb{K}, \\
\text { where } J(\mathbf{v})=\frac{1}{2} \mathbf{v}^{\top} \mathbf{K} \mathbf{v}-\mathbf{v}^{\top} \mathbf{f}+\sum_{i=1}^{n_{N}}-\mathcal{F} \mathbf{g}_{i}\left\|\mathbf{T}_{i}(\mathbf{v}-\mathbf{z})\right\|_{\mathbb{R}^{2}},
\end{array}\right\}
$$

where $\mathbf{K}$ and $\mathbf{f}$ is the stiffness matrix and right hand side, respectively. The matrices $\mathbf{N}$ and $\mathbf{T}$ describe mappings from $\mathbf{u}$ to $\left[\mathbf{u}_{N}\right],\left[\mathbf{u}_{T}\right]$, respectively. The vector $\mathbf{g}$ is the given slip bound,

$$
\mathbb{K}=\left\{\mathbf{v} \in \mathbb{R}^{n_{p}} \mid \mathbf{v}_{N}:=\mathbf{N} \mathbf{v} \leq \mathbf{c}_{N}\right\}
$$

is the cone of admissible displacements and $\mathbf{z}$ is the correction from the previous timestep solution. The gap between contact interfaces in the reference 
configuration we denote by $\mathbf{c}_{N}$. The numbers $n_{p}, n_{N}$ denote the displacement degrees of freedom count, the dual dof's (from the discretization of the duality pairing \langle\rangle ), respectively. Each of this matrices and vectors has a block structure according to the bodies of the multibody contact problem.

\section{Domain decomposition and dual formulation of the auxiliary Tresca friction problem}

Let mark all symbols from the previous section with $g$ as "global". At fig. 1 one can see the sketch of the domain decomposition of $i$ th body $\Omega_{i}^{g}$ into subdomains $\Omega_{i j}$. Let denote the space of the discretized displacements as $V^{g}$ and the decomposed space (broken space) by $V$. Dealing with the decomposed displacement $\mathbf{u}$ one have to enforce the continuity across the teared interface. This can be achieved by the "gluing" matrix $\mathbf{B}_{G}$ and the equality constraint imposed on $\mathbf{u}$ and in the same way can be handled the Dirichlet boundary condition

$$
\begin{aligned}
& \mathbf{u}^{g} \in V^{g} \Leftrightarrow(\mathbf{u} \in V) \wedge\left(\mathbf{B}_{G} \mathbf{u}=\mathbf{0}\right) \\
& \mathbf{u}^{g} \in K^{g} \Leftrightarrow(\mathbf{u} \in V) \wedge\left(\mathbf{B}_{G} \mathbf{u}=\mathbf{0}\right) \wedge\left(\mathbf{B}_{D} \mathbf{u}=\mathbf{0}\right) \Leftrightarrow(\mathbf{u} \in V) \wedge\left(\mathbf{B}_{E} \mathbf{u}=\mathbf{0}\right),
\end{aligned}
$$

where the matrix $\mathbf{B}_{E}$ is formed from the blocks $\mathbf{B}_{G}$ and $\mathbf{B}_{D}$. Substituting this into $\left(\mathcal{Q}_{A}^{i}\right)$ we obtain

$$
\left.\begin{array}{l}
\text { For given } \mathbf{g} \in \mathbb{R}_{-}^{n_{N}} \text { find } \mathbf{u} \in V \text { such that } \\
J(\mathbf{u}) \leq J(\mathbf{v}) \quad \forall \mathbf{v} \in \mathbb{K}, \text { subject to } \mathbf{B u}=\mathbf{B v}=\mathbf{0} \\
\text { where } J(\mathbf{v})=\frac{1}{2} \mathbf{v}^{\top} \mathbf{K} \mathbf{v}-\mathbf{v}^{\top} \mathbf{f}+\sum_{i=1}^{n_{N}}-\mathcal{F} \mathbf{g}_{i}\left\|\mathbf{T}_{i}(\mathbf{v}-\mathbf{z})\right\|_{\mathbb{R}^{2},}
\end{array}\right\}
$$

Let us mention that all the vectors and matrices have the block structure according to subdomains and so the computation with them is much more convenient. All the blocks in the stiffness matrix are positive semidefinite with the kernel of the dimension 6 in the case of 3D problems. Using the standard
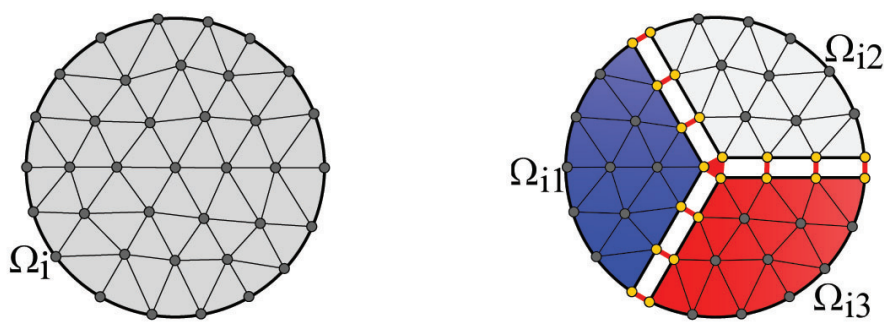

Figure 1: Original (left) and decomposed (right) domain. 
procedure to modify the non-differentiable term (see Dostál et al. [10]), we get

$$
\mathcal{F} \mathbf{g}_{i}\left\|\mathbf{T}_{i}(\mathbf{v}-\mathbf{z})\right\|=\max _{\left\|\boldsymbol{\lambda}_{T i}\right\|_{\mathbb{R}^{2}} \leq \mathcal{F} \mathbf{g}_{i}} \lambda_{T i}^{\top} \mathbf{T}_{i}(\mathbf{v}-\mathbf{z}) .
$$

Now, we can employ the additional Lagrange multipliers; $\lambda_{E}$ to enforce the equality condition, $\lambda_{N}$ to enforce the non-penetration condition and $\lambda_{T}$ that regularizes the non-differentiability. Finally we obtain the saddle point problem

$$
\left.\begin{array}{l}
\text { Find }\left(\mathbf{u}, \boldsymbol{\lambda}_{N}, \boldsymbol{\lambda}_{T}, \boldsymbol{\lambda}_{E}\right) \in \mathbb{R}^{n_{p}} \times \boldsymbol{\Lambda}(\mathbf{g}) \text { such that } \\
\mathcal{L}\left(\mathbf{u}, \boldsymbol{\lambda}_{N}, \boldsymbol{\lambda}_{T}, \boldsymbol{\lambda}_{E}\right)=\sup _{\substack{\boldsymbol{\lambda}_{N} \geq \mathbf{0} \\
\left\|\boldsymbol{\lambda}_{T}\right\|_{\mathbb{R}^{2}} \leq \mathcal{F} \mathbf{g}_{i} \\
\boldsymbol{\lambda}_{E} \in \mathbb{R}^{n_{E}}}} \inf _{\mathbf{v} \in \mathbb{R}^{n_{p}}} \mathcal{L}\left(\mathbf{v}, \boldsymbol{\mu}_{N}, \boldsymbol{\mu}_{T}, \boldsymbol{\mu}_{E}\right),
\end{array}\right\}
$$

where

$$
\begin{aligned}
& \mathcal{L}\left(\mathbf{v}, \boldsymbol{\mu}_{N}, \boldsymbol{\mu}_{T}, \boldsymbol{\mu}_{E}\right)=\frac{1}{2} \mathbf{v}^{\top} \mathbf{K} \mathbf{v}-\mathbf{v}^{\top} \mathbf{f}+ \\
& +\boldsymbol{\mu}_{T}^{\top}(\mathbf{T} \mathbf{v}-\mathbf{T z})+\boldsymbol{\mu}_{N}^{\top}\left(\mathbf{N} \mathbf{v}-\mathbf{c}_{N}\right)+\boldsymbol{\mu}_{E}^{\top}\left(\mathbf{B}_{E} \mathbf{v}-\mathbf{c}_{E}\right) \\
& \boldsymbol{\Lambda}(\mathbf{g})=\mathbb{R}_{+}^{n_{N}} \times\left\{\boldsymbol{\mu}_{T} \in \mathbb{R}^{2 n_{N}} \mid\left\|\boldsymbol{\lambda}_{T i}\right\|_{\mathbb{R}^{2}} \leq \mathcal{F} \mathbf{g}_{i}\right\} \times \mathbb{R}^{n_{E}} \text {. }
\end{aligned}
$$

After small change of notation to group the indices $N, T, E$ together, swapping the supremum and infimum, eliminating of displacement using the pseudoinverse matrix $\mathbb{K}^{+}$and the kernel matrix $\mathbf{R}$ (see all this steps in Dostál et al. [2]) we obtain the dual formulation

$$
\text { Find } \boldsymbol{\lambda} \in \boldsymbol{\Lambda}^{e q}(\mathbf{g}) \text { such that } \mathcal{S}(\boldsymbol{\lambda}) \leq \mathcal{S}(\boldsymbol{\mu}) \quad \forall \boldsymbol{\mu} \in \boldsymbol{\Lambda}^{e q}(\mathbf{g})
$$

where

$$
\begin{aligned}
& \mathcal{S}(\mu)=\frac{1}{2} \lambda^{\top} \overbrace{\mathbf{B K}^{\dagger} \mathbf{B}^{\top}}^{\mathbf{F}} \lambda-\lambda^{\top} \overbrace{\mathbf{B K}^{\dagger} \mathbf{f}-\mathbf{C}}^{\mathbf{d}} \\
& \boldsymbol{\Lambda}^{e q}(\mathbf{g})=\{\boldsymbol{\lambda} \in \boldsymbol{\Lambda}(\mathbf{g}) \mid \underbrace{\mathbf{R}^{\top} \mathbf{B}^{\top}}_{\mathbf{G}} \boldsymbol{\lambda}=\underbrace{\mathbf{R}^{\top} \mathbf{f}}_{\mathbf{e}}\}
\end{aligned}
$$

\section{Optimality}

After homogenization of the equality constraint in (4) and returning back to the same notation one can enhance the resulting problem by putting the projectors from the homogenized equality constraints, which results in the problem

$$
\text { Find } \boldsymbol{\lambda} \in \boldsymbol{\Lambda}^{\star}(\mathbf{g}) \text { such that } \mathcal{S}^{\star}(\boldsymbol{\lambda}) \leq \mathcal{S}^{\star}(\boldsymbol{\mu}) \quad \forall \boldsymbol{\mu} \in \boldsymbol{\Lambda}^{\star}(\mathbf{g}) \text {, }
$$

where

$$
\begin{aligned}
\mathcal{S}^{\star}(\boldsymbol{\mu}) & =\frac{1}{2} \boldsymbol{\lambda}^{\top}(\mathbf{P F P}+\rho \mathbf{Q}) \boldsymbol{\lambda}-\boldsymbol{\lambda}^{\top} \mathbf{P d} \\
\boldsymbol{\Lambda}^{\star}(\mathbf{g}) & =\{\boldsymbol{\lambda} \in \boldsymbol{\Lambda}(\mathbf{g}) \mid \mathbf{G} \boldsymbol{\lambda}=\mathbf{0}\}, \quad \mathbf{Q}=\mathbf{G}^{\top}\left(\mathbf{G} \mathbf{G}^{\top}\right)^{-1} \mathbf{G}, \quad \mathbf{P}=\mathbf{I}-\mathbf{Q} .
\end{aligned}
$$


A good choice of the regularization parameter is given by $\bar{\rho}=\|P F P\|$, as this is the largest value for which

$$
\|\mathbf{P F P}\| \geq\|\mathbf{P F P}+\rho \mathbf{Q}\|
$$

The classical estimates by Farhat et al. [4] of the extreme eigenvalues says that if the decompositions and the discretizations are sufficiently regular, then there exists constants $C_{1}>0$ and $C_{2}>0$ independent of the discretization parameter $h$ and the decomposition parameter $H$ such that

$$
C_{1} \frac{h}{H} \leq \lambda_{\min }(\mathbf{P F P} \mid \operatorname{ImP}) \quad \text { and } \quad \lambda_{\max }(\mathbf{P F P} \mid \operatorname{ImP})=\|\mathbf{P F P}\| \leq C_{2},
$$

where $\lambda_{\min }$ and $\lambda_{\max }$ denote the extremal eigenvalues of the corresponding matrices.

For the solution of 5 we propose to use SMALSE (semi-monotonic augmented Lagrangian method for separable and equality constraints) Dostál and Kozubek [5]. SMALSE enforces the equality constraints by the Lagrange multipliers generated in the outer loop, while the auxiliary inner quadratic programming problems with separable inequality constraints but without equality constraints are solved approximately in the inner loop by the MPGP algorithm proposed by Dostál and Kozubek [5].

The unique feature of combination of SMALSE with MPGP for solving the quadratic programming problems (5) that arise from the auxiliary Tresca friction problems is the bound on the number of iterations whose cost is proportional to the number of variables, so that it can return an approximate solution for the cost proportional to the number of variables.

\section{Numerical experiments}

In this section we want to test the previously mentioned algorithms to show their optimality. The algorithms were implemented into our MatSol software (Kozubek et al. [11]) and tested on academic and real world problems.

\subsection{Structured grid: Two cantilever beams example}

We shall consider two elastic bodies represented by the bricks $\Omega_{i}$ made of a homogenous and isotropic material which are characterized by Young's modulus $E_{i}$ and Poisson's ratios $\sigma_{i}, i=1, \ldots, 2$ :

$$
\begin{aligned}
& \Omega_{1}=(0,2) \times(-0.05,1.05) \times(-1,0), \quad E_{1}=30000 \mathrm{MPa}, \sigma_{1}=0.27 \\
& \Omega_{2}=\left(\frac{2}{3}, \frac{8}{3}\right) \times(0,1) \times(0.1,1.1), \quad E_{2}=30000 \mathrm{MPa}, \sigma_{2}=0.35 .
\end{aligned}
$$

The bodies are subjected by the surface tractions $P_{i U} \cdot \phi_{a}(t), P_{i L} \cdot \phi_{b}(t)$ as is depicted in fig. 2. The functions $\phi_{a}, \phi_{a}$ (fig. 3) characterizes the history of the 


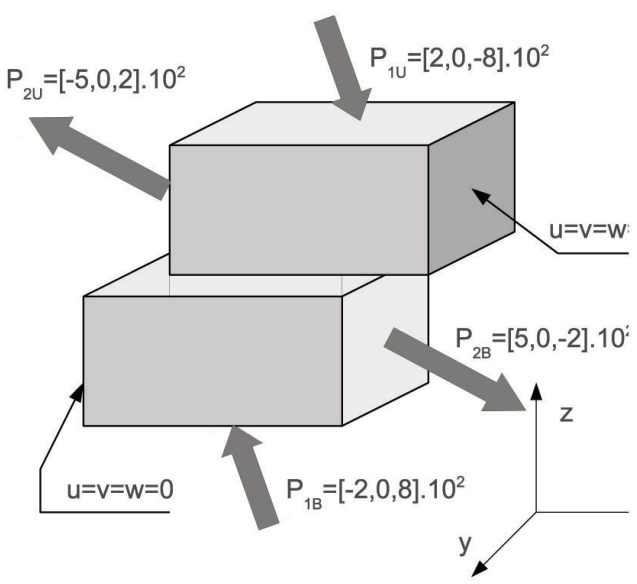

Figure 2: Geometry of cantilever beams.

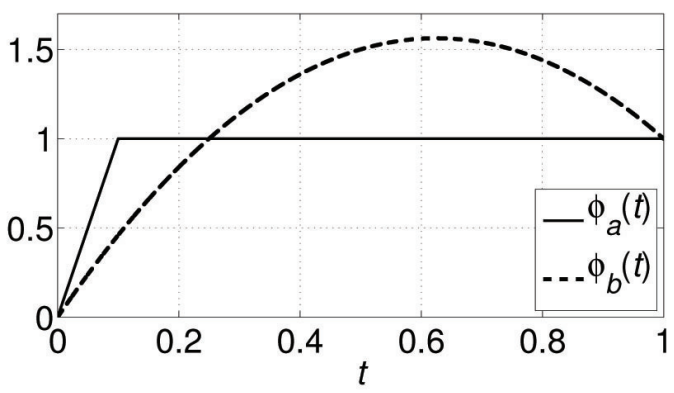

Figure 3: Geometry of cantilever beams.

loading process. We used structured trilinear grid with varying discretizations. The discretization and decomposition parameters were properly chosen to keep the ratio $H / h$ constant. The computation was done with the Coulomb friction coefficient $\mathcal{F}=0.5$ and for 10 timesteps.

The resulting von Mises stress in timesteps 5 and 10 are depicted in figures 4 and 5. The characteristics of computation are summarized in table 1. We can see that the number of matrix-vector multiplications and SMALSE inner iterations increases with the increasing problem size. The number of inner iterations increases due to the use of Coulomb friction boundary condition. In addition, if we take into account the fact that the first approximately ten iterations of the SMALSE algorithm (in each timestep) are inexpensive to Hessian multiplications then one can see that the ratio of Hessian multiplication per inner iteration varies only a little, as was predicted by the theory. 
Table 1: Numerical characteristics of 3D cantilever beams example.

\begin{tabular}{lrrrr}
\hline Number of subdomains & 32 & 108 & 256 & 500 \\
Number of CPUs & 20 & 20 & 20 & 20 \\
Primal variables & 127,775 & 431,244 & $1,022,208$ & $1,996,500$ \\
Dual variables & 23,763 & 89,283 & 222,462 & 446,916 \\
Null space dimension & 192 & 648 & 1536 & 3000 \\
SMALSE-M iterations & 123 & 125 & 136 & 147 \\
Hessian multiplications & $\mathbf{1 8 3 4}$ & $\mathbf{2 1 6 0}$ & $\mathbf{2 5 2 2}$ & $\mathbf{3 0 9 9}$ \\
Solution time [s] & 197 & 607 & 1813 & 4446 \\
Total time [s] & 302 & 1181 & 3586 & 9301 \\
\hline
\end{tabular}

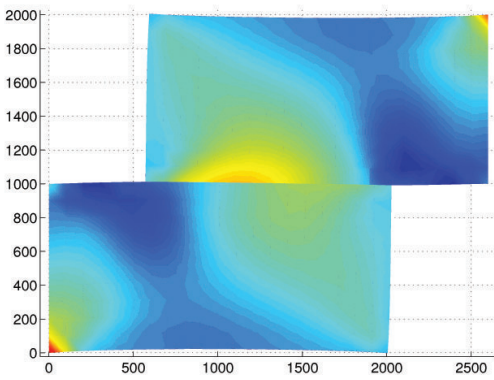

Figure 4: two beams, $t_{s}=5$.

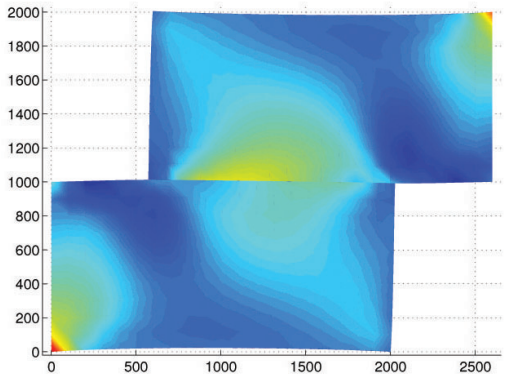

Figure 5: two beams, $t_{s}=10$.

\subsection{Real world problem application: yielding clamp connection}

To show that our approach is capable also to solve real world contact problems we consider the analysis of the yielding clamp connection of steel arched supports depicted in fig. 6. This type of construction is used to support the mining openings. It is a typical multibody contact, where the yielding connection plays the role of the mechanical protection against destruction, i.e., against the total deformation of the supporting arches. We consider again the quasistatic Coulomb friction problem contact with $\mathcal{F}=0.5$ and 10 timesteps. The problem was decomposed by METIS into 150 subdomains and discretized by 520,833 and 102,724 primal and dual variables, respectively. The total displacements for timesteps 6 and 10 are depicted in fig. 6 . The computation required 8278 matrix-vector multiplications. The solution time was $2315 \mathrm{~s}$ and the total time $4293 \mathrm{~s}$. 

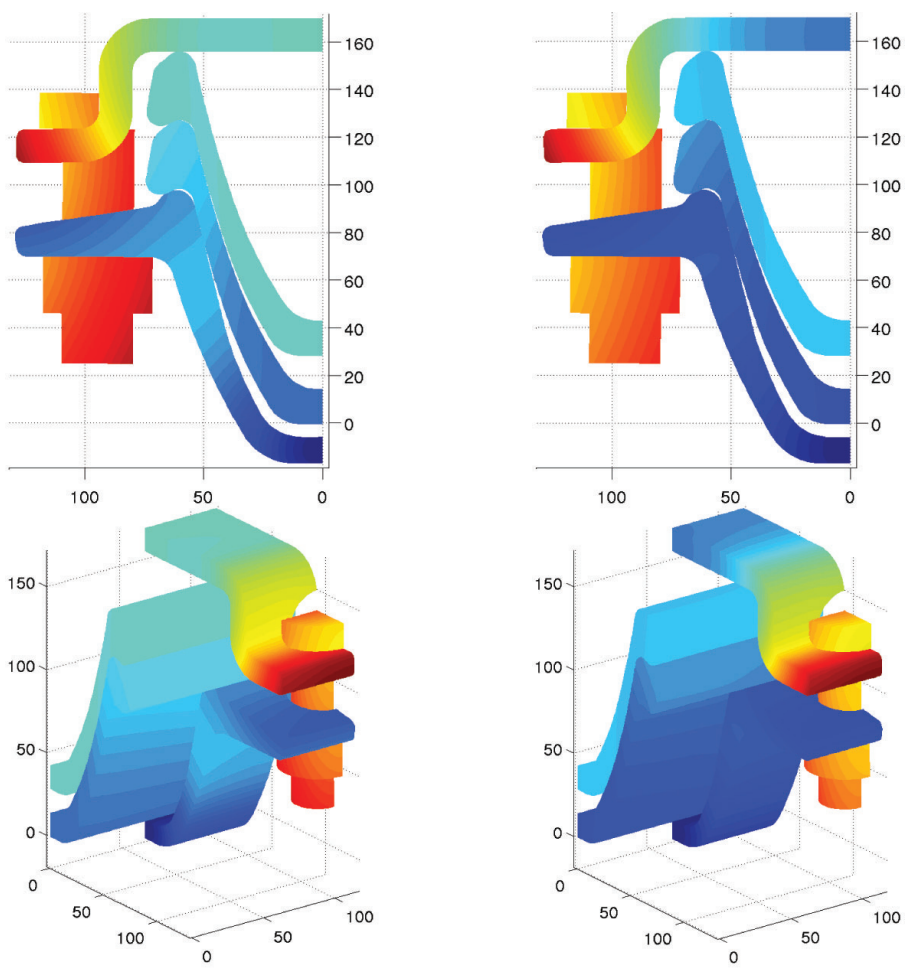

Figure 6: Steel support, displacement for $t_{s}=6$ (left) and $t_{s}=10$ (right).

\section{Acknowledgements}

This proceeding has been elaborated in the framework of the IT4Innovations Centre of Excellence project, reg. no. CZ.1.05/1.1.00/02.0070 supported by Operational Programme 'Research and Development for Innovations' funded by Structural Funds of the European Union and state budget of the Czech Republic. This work was also supported by research project MSM6198910027.

\section{References}

[1] Eck, C., Jarušek, J. \& Krbec, M., Unilateral contact problems: Variational methods and existence theorems. Taylor and Francis, 2005.

[2] Dostál, Z., Horák, D. \& Kučera, R., Total FETI - an easier implementable variant of the FETI method for numerical solution of elliptic PDE. Commun Numer Methods in Engrg, 22, pp. 1155-1162, 2006.

[3] Dostál, Z., Kozubek, T., Brzobohatý, T., Markopoulos, A. \& Vlach, O., Scalable TFETI with preconditioning by conjugate projector for transient frictionless contact problems of elasticity.

[4] Farhat., C., Mandel, J. \& Roux, F., Optimal convergence properties of the 
FETI domain decomposition method. Comput Methods Appl Mech Engrg, 115, pp. 365-385, 1994.

[5] Dostál, Z. \& Kozubek, T., An optimal algorithm and superrelaxation for minimization of a quadratic function subject to separable convex constraints with applications. Mathematical Programming, pp. 1-26, 2011.

[6] Dostál, Z. \& Kučera, R., An optimal algorithm for minimization of quadratic functions with bounded spectrum subject to separable convex inequality and linear equality constraints. SIAM Journal on Optimization, 20(6), pp. 29132938, 2010.

[7] Haslinger, J., Vlach, O., Dostál, Z. \& Banitopoulos, C., The FETI-DP domain decomposition method for quasistatic contact problems with coulomb friction. Variational Formulations in Mechanics: Theory and Applications CIMNE Barcelona, pp. 125-141, 2007.

[8] Haslinger, J., Kuera, R., Vlach, O. \& Baniotopoulos, C., Approximation and numerical realization of $3 \mathrm{~d}$ quasistatic contact problems with coulomb friction. Mathematics and Computers in Simulation, 2011.

[9] Rocca, R. \& Coccu, M., Numerical analysis of quasistatic unilateral contact problems with local friction. SIAM J Numer Anal, 39, pp. 1324-1342, 2001.

[10] Dostál, Z., Kozubek, T., Markopoulos, A., Brzobohatý, T., Vondrák, V. \& Horyl, P., A theoretically supported scalable tfeti algorithm for the solution of multibody $3 \mathrm{~d}$ contact problems with friction, 2011. In Press.

[11] Kozubek, T., Markopoulos, A., Brzobohatý, T., Kučera, R., Vondrák, V. \& Dostál, Z., Matsol - matlab efficient solvers for problems in engineering. "http://matsol.vsb.cz/", 2009. 